Instituto Internacional de Investigación y Desarrollo Tecnológico Educativo INDTEC, C.A.

DOI: https://doi.org/10.29394/Scientific.issn.2542-2987.2019.4.E.8.136-152

OAI-PMH: http://www.indteca.com/ojs/index.php/Revista Scientific/oai

Artículo Original / Original Article

\title{
Reflexión de la gestión administrativa para mejorar los resultados académicos de la Comuna de Ovalle
}

$\begin{array}{r}\text { Autores: Natalie del Carmen Muñoz Morales } \\ \text { Universidad Miguel de Cervantes, UMC } \\ \text { natalie.munoz@profe.umcervantes.cl }\end{array}$
Talca, Chile
http://orcid.org/0000-0002-5430-1517
Albino Enzon González González
Universidad Miguel de Cervantes, UMC
albino.gonzalez@profe.umcervantes.cl
Talca, Chile
https://orcid.org/0000-0003-1574-5390

\section{Resumen}

En la actualidad existen diversas investigaciones que se dirigen a encontrar explicaciones y factores, que influyen en el bajo o alto rendimiento de los estudiantes, estudios descriptivos, explicativos, exploratorios y correlacionales. El presente artículo tiene como propósito comprender cómo procesos y procedimientos de la gestión administrativa de un establecimiento educacional municipal de alta vulnerabilidad intervienen en el logro académico de los/las alumnos/as de la comuna de Ovalle, durante el año 2008-2009, en el marco del programa de capacitación a equipos directivos y docentes de escuelas vulnerables de la región de Coquimbo. Para ello se aborda diversos aspectos teóricos necesarios de conocer para lograr una comprensión integral de las dinámicas educativas centradas en la gestión administrativa de los establecimientos educacionales que han sido foco de esta investigación. La investigación es de tipo cualitativa, a partir de la cual se hace un análisis de contenido expresado de manera descriptiva. Cabe destacar que, las conclusiones permitieron promover diferentes acciones como: sistematización, conducción e involucramiento de todos los actores de la Unidad Educativa. Sin embargo, evidenciaron resultados dispares en la evaluación de los aprendizajes realizados con posterioridad a los/las alumnos/as.

Palabras clave: gestión; educación; rendimiento académico.

Cómo citar este artículo:

Muñoz, N., \& González, A. (2019). Reflexión de la gestión administrativa para mejorar los resultados académicos de la Comuna de Ovalle. Revista Scientific, 4(Ed. Esp.), 136-152, e-ISSN: 2542-2987. Recuperado de: https://doi.org/10.29394/10.29394/Scientific.issn.2542-2987.2019.4.E.8.136-152

Fecha de Recepción: 09-05-2019
Fecha de Aceptación: 16-07-2019
Fecha de Publicación: 05-09-2019 


\title{
Reflection of administrative management to improve the academic results of the Commune of Ovalle
}

\begin{abstract}
At present there are several investigations that are aimed at finding explanations and factors that influence the low or high performance of students, descriptive, explanatory, exploratory and correlational studies. The purpose of this article is to understand how processes and procedures of the administrative management of a high-vulnerability municipal educational institution intervene in the academic achievement of the students of the Ovalle commune, during the year 2008-2009, in the framework of the training program for management teams and teachers of vulnerable schools in the Coquimbo region. To this end, various theoretical aspects are necessary to know to achieve a comprehensive understanding of the educational dynamics focused on the administrative management of educational establishments that have been the focus of this research. The research is of a qualitative type, from which a content analysis expressed in a descriptive manner is made. It should be noted that, the conclusions allowed to promote different actions such as: systematization, conduction and involvement of all the actors of the Educational Unit. However, they showed disparate results in the evaluation of the lessons learned after the students.
\end{abstract}

Keywords: management; education; academic achievement.

How to cite this article:

Muñoz, N., \& González, A. (2019). Reflection of administrative management to improve the academic results of the Commune of Ovalle. Revista Scientific, 4(Ed. Esp.), 136-152, e-ISSN: 25422987. Recovered from: https://doi.org/10.29394/10.29394/Scientific.issn.2542-2987.2019.4.E.8.136-152

Date Received:

09-05-2019
Date Acceptance:

16-07-2019
Date Publication: 05-09-2019 


\section{Introducción}

Como punto de partida, se tiene que Rojas (2019), indica que:

El docente debe trazarse objetivos de cómo motivar al alumno a que se incorpore a su clase, qué estrategias educativas e innovadoras puede aplicar para que desde su praxis el docente impulse al alumno para que este mejore su rendimiento y nivel académico (pág. 129).

Por tal motivo, la educación desde un punto de vista general, es un tema de análisis, debate y propuestas, más aún cuando se habla de los aprendizajes de los estudiantes, en los que cada año posterior a la rendición de la prueba del Sistema de Medición de la Calidad de la Educación (SIMCE), aparece en los medios de comunicación masiva, distintos personeros hablando y reflexionando acerca de la mejor manera de hacer que la educación del país mejore.

Es entonces, en este contexto que se plantea una inquietud de comprender cómo procesos y procedimientos de la gestión administrativa de calidad de un establecimiento educacional municipal de alta vulnerabilidad, intervienen en el logro académico de los/las alumnos/as de la comuna de Ovalle, durante los años 2008-2009, mediante el análisis de estos procesos y procedimientos implementados y obtenidos durante el mismo período, por docentes directivos de los establecimientos educacionales municipales con alta vulnerabilidad de la Comuna de Ovalle a partir de la implementación del programa de capacitación a equipos directivos y docentes de escuelas vulnerables de la región de Coquimbo durante el año 2008-2009, realizada por la Fundación especializada en Servicios de Formación y Desarrollo Profesional Docente (SEPEC), con el fin de precisar los aspectos intervinientes en la eficacia de la gestión administrativa que influyen en el ascenso de los resultados académicos de los/las alumnos/as.

El estudio se inicia con una visita a los establecimientos focalizados 
donde se recaba información respecto de la constitución orgánica de cada uno de ellos. Se realizan entrevistas y aplican cuestionarios a directivos.

$\mathrm{Si}$ bien resulta difícil revisar toda la literatura para localizar investigaciones específicas que describan o expliquen la naturaleza de las variables asociadas al éxito o fracaso académico del punto de vista de la gestión administrativa de equipos directivos, se reconoce como una dimensión que forma parte de un todo y que por sí misma es muy importante.

Las causas identificadas se han clasificado según las dimensiones propuestas por Weiner (1985), citado por Valle, Raiano, García, Suligoy y Gómez (2014):

En ámbitos académicos, se aplica esta teoría cuando los estudiantes aluden a los factores causales que explicarían sus resultados académicos. Las atribuciones son analizadas según el rendimiento académico de los estudiantes, de modo que se puede apreciar la distinta valoración que unos y otros realizan. Se combina el análisis cualitativo con datos cuantitativos obtenidos de la aplicación de un cuestionario. Se analizan las variables: significado de éxito y significado de fracaso (pág. 139).

Se considera necesario y enriquecedor sustentar una investigación de esta naturaleza con acervo teórico y bibliográfico pertinente, para lo cual se describen a continuación algunas investigaciones realizadas a nivel nacional e internacional.

En Chile las Escuelas Efectivas plantean que los estudiantes se concentran de acuerdo a sus condiciones de pobreza, han mostrado resultados de aprendizaje de excelencia.

La gestión institucional centrada en lo pedagógico, como lo señala el estudio, destaca en aquellas escuelas que trabajan en condiciones de pobreza, las que son capaces de tomar decisiones como: planificación institucional en torno al aprendizaje de los alumnos, uso de materiales, asignación de profesores a los cursos, entre otros. Donde todo está planificado 
y se va evaluando lo que se hace. Por ende, el trabajo de una escuela efectiva supone siempre una buena gestión institucional pero alineada con un trabajo de excelencia en la sala de clases. Además, dice que las escuelas que decaen en el logro de los aprendizajes de sus estudiantes son aquellas en las cuales a veces disminuye el liderazgo del director, los profesores dejan de planificar o no se adaptan a los cambios y no tienen la capacidad para evaluarse constantemente.

El programa capacitación a directivos y docentes de escuelas vulnerables región de Coquimbo, comenzó con la aplicación de pruebas en los subsectores de educación matemática, lenguaje y comunicación y comprensión del medio natural, social y cultural. Los resultados se entregaron en forma detallada, por estudiante; lo que permitió una intervención más eficaz desde la gestión curricular, como también desde la gestión administrativa, que permitió a equipos de docentes directivos elaborar procesos y procedimientos con el objetivo de elevar los resultados obtenidos en el diagnóstico realizado a niños y niñas de primer ciclo básico. Los resultados evidenciaron que los aprendizajes más descendidos se concentraban en el subsector de educación matemática.

Cabe destacar, según los autores Fernández, Toledo, Martínez, Casado, Navarro y Folgado (2018), que:

La lucha para una igualdad efectiva entre hombres y mujeres debe estar basada en un sistema educativo que trasmita valores igualitarios y elimine los estereotipos de género. Si bien, la práctica de la pedagogía coeducativa se ha centrado principalmente en los niveles inferiores de la enseñanza, la docencia universitaria puede y debe también jugar un papel importante en este sentido y contribuir al avance en las políticas de igualdad (pág. 1832).

El tema elegido para el artículo está directamente relacionado con evaluación desde la perspectiva de resultados académicos. En concordancia 
con el enfoque actual que vincula calidad de la educación, con resultados de los aprendizajes de los/las estudiantes, obtenidos especialmente en evaluaciones de aplicación nacional por el SIMCE, por lo que, aunque se puede cuestionar el método, hasta ahora en el país no existe otro sistema para medir lo que los estudiantes aprenden y, es lo que mueve al estado a invertir recursos financieros para proveer a las escuelas de la implementación necesaria para que los niños/as aprendan más y en mejores condiciones.

En Chile se han realizado muchos intentos por mejorar los rendimientos académicos de los/las estudiantes por lo que se han implementado diversas reformas y múltiples programas que han modificado y enriquecido el currículum, sin embargo, no es suficiente la sola instalación de ellos en las escuelas, para lograr cambios u objetivos esperados.

\section{Metodología}

La investigación fue desarrollada durante el primer semestre del año 2009, en tres escuelas de la comuna de Ovalle, que formaron parte del programa capacitación a directivos y docentes de escuelas vulnerables región de Coquimbo, y que obtuvieron bajos resultados en el subsector de educación matemática año 2007.

La metodología utilizada se encuentra enmarcada dentro de una investigación cualitativa del tipo descriptiva, no experimental, transaccional, se ha considerado que el paradigma adecuado a la investigación es el descriptivo interpretativo, puesto que busca entender, caracterizar y describir fenómenos sociales considerando cualidades desde la propia perspectiva de los actores. Es transaccional porque estudia en profundidad una situación concreta, desarrolla hipótesis que se dan en casos individuales.

\section{Resultados}

Para dar paso a los resultados, se debe conocer un poco algunos 
aspectos relevantes que permiten el sustento teórico de las conclusiones, las cuales atienden a aspectos diversos de la vida escolar, en el entendido que las relaciones y fines de un sistema escolar debe abarcar al ser humano en toda su dimensión tanto física, cognitiva, afectiva, espiritual, social, entre otros, expresado en la tabla 1. Del mismo modo, considerar en forma efectiva la diversidad de actores que intervienen en el proceso que permite tener una mirada más enriquecedora que mejorará la toma de decisiones.

Tabla 1. Aspectos y dimensiones de la vida y sistema escolar.

\begin{tabular}{|c|l|}
\hline & $\begin{array}{l}\text { La confianza en sí mismo y el desarrollo de su } \\
\text { personalidad. } \\
\text { El manejo de la ansiedad. } \\
\text { La autonomía para que asuma el control de su vida } \\
\text { y aproveche sus potencialidades. } \\
\text { La aceptación de reglas, límites y prohibiciones. } \\
\text { educación en la vida escolar. } \\
\text { El reconocimiento del otro. } \\
\text { La participación en el bien común. } \\
\text { El trabajo como oportunidad para darle sentido a } \\
\text { la vida. } \\
\text { sistema escolar. }\end{array}$ \\
$\begin{array}{c}\text { La amistad y el amor verdadero que son la base } \\
\text { de una sana convivencia. } \\
\text { La reflexión para que él defina su posición ante la } \\
\text { existencia. }\end{array}$ \\
\hline $\begin{array}{l}\text { Física, cognitiva, afectiva, espiritual, social, entre } \\
\text { otros. }\end{array}$ \\
\hline
\end{tabular}

Fuente: Espinoza (2009); adaptación de Los Autores (2019).

Juntamente a lo anterior, existen énfasis que han de destacarse como es el caso del ámbito curricular, donde el énfasis curricular de estas escuelas, radica en formar estudiantes que sean valorados(as) como integrantes de una 
sociedad democrática y pluralista, en el ámbito de la convivencia escolar esta la asimilación de normas de conducta y formación de hábitos, donde los estudiantes acepten y practiquen valores que les permitan integrarse al mundo con metas y propósitos definidos, que amen a sus familias, su comunidad y a sí mismos(as).

Para aproximarse a las formas de gestión y la realidad escolar se clasifica en distintas dimensiones, una de ellas es la gestión administrativa, desde el punto de vista analítico es una importante herramienta que permite observar, criticar y observar lo que pudiese suceder al interior de un establecimiento educacional.

Además, Chica (2015), citado por Cerdas, García, Torres y Fallas (2017), en este sentido:

Aclara que la gestión educativa busca aplicar los principios generales de la gestión, que han estado presentes en la teoría de la administración y se extrapolan al campo específico de la educación. El objeto de la gestión educativa como disciplina es el estudio de la organización del trabajo en el campo de la educación, por tanto, está influenciada por teorías de la administración, pero además existen otras disciplinas que han permitido enriquecer el análisis, como son "la administración, la filosofía, las ciencias sociales, la psicología, la sociología y la antropología" (pág. 101).

Es importante también mencionar la gestión administrativa escolar, ya que este concepto está adquiriendo cada vez mayor importancia, y está formulado desde el ministerio de educación para que se entreguen capacitaciones y articulen acciones que potencien la gestión desde distintas áreas de la Educación. Una muestra de ello es el fondo de mejoramiento de la gestión educacional que posee recursos propios para mejorar aspectos vinculados directamente a la gestión y que sería imposible cubrirlos con excedentes de recursos financieros, en algunos casos, dado el desfinanciamiento de la educación municipalizada. 
En este sentido, cuando se hace referencia la administración educacional se encuentran determinadas funciones administrativas, que hacen que sea eficaz el proceso de administrar. Ellas son: planificación, organización, dirección, control y evaluación. El sistema educacional chileno se caracteriza, desde el punto de vista de la organización en general, por ajustarse a un modelo de sistema de administración del tipo clásico, puesto que posee una estructura organizada-jerarquizada.

La escuela constituye también un sistema, donde el conjunto de personas que la integran (directivos, técnicos, docentes, padres y apoderados, alumnos, entre otros), se relacionan y trabajan en conjunto para lograr objetivos comunes y determinados. (2019):

Desde esta perspectiva, según Cárdenas (2017), citado por López

La educación no es dar una clase o realizar una actividad, la educación es formar ciudadanos para el hoy y para el mañana y que un maestro desde sus prácticas es capaz de cambiar la realidad; comprendí que educar es dejar huellas en cada una de las personas que hacen parte de nuestro recorrido como docentes y que para lograr esto se necesita amor por lo que se hace, paciencia y mucha dedicación (pág. 250).

De acuerdo a lo planteado, los directivos, técnicos, docentes, administrativos, alumnos, apoderados, etc. son subsistemas de una organización educativa, los cuales cumplen con diversas funciones y actividades específicas, las que están orientadas a un fin último.

El actual modelo de educación que se está dando en Chile, tiene relación con importantes avances a nivel de cobertura escolar y que está viendo la posibilidad de fortalecer una educación de calidad para todos los estudiantes chilenos, otorgándoles la oportunidad de lograr mejores y mayores aprendizajes.

El análisis de contenido permitió indagar en las notas de campo, 
interpretar la información que serviría para la investigación. Su utilización permitió relacionar los hallazgos encontrados en el trabajo de campo con la argumentación teórica. Por otra parte, en las notas de campo, para comprender e interpretar la realidad como fue entendida por los sujetos participantes en los escenarios estudiados.

Es evidente que todos los equipos directivos plantearon procesos similares, con énfasis diferentes, sin embargo solo en un establecimiento educacional elevó significativamente los resultados académicos, lo cual deja de manifiesto que elevar los resultados académicos, no depende de una estrategia específica, sino del grupo humano que dirige la escuela, lo que tiene gran importancia, y no tiene que ver únicamente con capacidades profesionales, sino de características personales y criterios de administración. Lo que hace más complejo la formación de equipos directivos que cumplan con el propósito de elevar el nivel de aprendizaje de los/las estudiantes.

Es de cuestionarse también, que no es suficiente darle importancia a un determinado grupo de procedimientos, sino contar con los tiempos y las personas adecuadas para realizar cada una de sus funciones dentro del plan de trabajo, caso contrario, aunque el mejor procedimiento se puede desvirtuar en el proceso.

En los diferentes trabajos de investigación y propuestas gubernamentales se plantea la importancia de la labor del docente en el aula, en este caso se planteó la influencia de los procesos y procedimientos del equipo directivo con foco en los aprendizajes de los estudiantes. Son los docentes directivos quienes tienen la capacidad, la responsabilidad de administrar en función de mejorar los aprendizajes de los estudiantes y no en relación a preferencias personales o criterios poco profesionales, junto a tomar decisiones respecto de todos los ámbitos educativos dentro de una escuela.

De acuerdo a lo anterior, queda de manifiesto que el equipo directivo que trabajó de manera sistemática y traspasó todas las áreas y/o dimensiones, 
fue el que logró elevar significativamente los resultados académicos de sus estudiantes. Los demás equipos directivos, procuraron trabajar con el recurso humano que tenían, haciendo lo mejor que podían, no interviniendo el recurso humano para no herir susceptibilidades, lo que trajo el costo que los alumnos aprendieran menos.

En este contexto, la actual legislación deja muy poco margen a los sostenedores de escuelas municipales de gestionar o establecer acciones que fortalezcan los equipos técnicos y/o directivos de los establecimientos para favorecer los aprendizajes de los/las estudiantes, más aún se consideran circunstancias políticas imperantes del momento, que no ayudan a avanzar en los procesos y procedimientos eficaces implementados por determinados equipos directivos, poniendo término, en muchas ocasiones, a un proceso establecido quedando en un total abandono y discontinuidad y que conlleva a la consecuencia de desigualdad e inequidad de los aprendizajes de los/las alumnos/as.

\section{Conclusión}

Se puede comprender cómo los procesos y procedimientos de la gestión administrativa de calidad de un establecimiento educacional municipal de alta vulnerabilidad interviene en el logro académico de los/las alumnos/as de la ciudad de Ovalle, como también de los resultados disimiles que obtuvieron los/las estudiantes de primer ciclo de enseñanza básica, después que los docentes y directivos fueron capacitados por el Programa de Escuela Vulnerables de la Región de Coquimbo.

La contribución de este estudio se encuentra asociada al Sistema de Aseguramiento de Calidad de la Gestión (SACGE) área Liderazgo, dimensión: conducción. Porque el estudio concluyó que es necesario, para el ascenso de los resultados de los/las alumnos, un equipo directivo con fortalezas en aspectos técnicos curriculares y de liderazgo reconocido y valorado por todos 
los integrantes de la Unidad Educativa.

Cabe destacar, como aspecto de modelo de gestión mencionado en el marco teórico, las escuelas objeto de esta investigación, tienen en común una organización, una estructura y funciones asociadas al cumplimiento de un propósito que trata de comprender y orientar los esfuerzos de la organización educativa en la búsqueda de los objetivos.

De igual forma, se pudo constatar que la escuela que obtuvo ascensos significativos en los resultados de los/las estudiantes, su organización, su estructura y funciones estuvieron asociadas al cumplimiento del propósito de mejorar los resultados de los aprendizajes de los/las estudiantes, llevando a la práctica los conocimientos adquiridos en la capacitación otorgada por la Fundación SEPEC, a diferencia de las otras dos escuelas que sus funciones y esfuerzos se destinaron a la gestión administrativa con énfasis diferentes y que no apuntaba al aprendizaje de los/las estudiantes dentro del aula.

Además, el análisis y la reflexión de los aportes teóricos y hallazgos recopilados de los establecimientos educacionales o permite señalar que a partir del programa de capacitación para directivos y docentes de escuelas vulnerables de la región de Coquimbo otorgado por la Fundación SEPEC, la escuela (1) que implementó procesos y procedimientos en el primer ciclo básico, de manera sistemática y en donde existió una planificación, formalización y evaluación de dichos procesos y procedimientos, aspectos de la gestión administrativa, elevó sus resultados de manera significativa (más de 10 puntos) en todos los subsectores evaluados (lenguaje y comunicación, educación matemática, comprensión del medio natural, social y cultural.

También se tuvo conclusión que los procesos y procedimientos implementados por esta escuela (escuela 1) abarcan los tres ámbitos (Gestión Administrativa, Pedagógica y Financiera) considerados, relevantes, para el logro de los aprendizajes de los alumnos en la escuela, como lo establece el estudio ¿Quién dijo que no se puede?. la forma en cómo se establece la 
dinámica educativa en función de la gestión administrativa y su influencia en los aprendizajes de los/las estudiantes. Lo que viene a responder la conjetura planteada en esta investigación.

Cabe señalar además, que un aspecto importante que resalta, a la luz del análisis de las entrevistas, es que existen respuestas contradictorias entre docentes directivos del mismo establecimiento respecto de algunos aspectos de los procesos y procedimientos aplicados en los establecimientos educacionales en los cuales desempeñan funciones.

Otro aspecto a considerar en los procesos y procedimientos de la gestión administrativa, es que en la Escuela 1 gran número de docentes se acogió a jubilación, situación que dio la oportunidad de ingreso a docentes recién egresados con quienes la relación de liderazgo, desde el equipo directivo, se desarrolló de manera más fluida permitiendo que estos procesos y procedimientos planteados fueran concretados de manera asertiva, entendiéndose por ello que existió una estructura organizacional integrada por nuevos actores.

Por otro lado, la característica central que tuvo la implementación de procesos y procedimientos de la gestión administrativa, guarda directa relación con las acciones concretas implementadas por los docentes dentro del aula y el apoyo de los directivos en su accionar, con lo que se llega a establecer que uno de los factores más incidentes al momento de tener mejores resultados en los aprendizajes de los/as estudiantes, está centrado en lo pedagógico, dentro de la escuela, lo que provoca el cambio significativo en los resultados.

Se concluye, que si los directivos de una unidad educativa trabajan en forma cohesionada, focalizan sus esfuerzos, establecen procesos y procedimientos en base a un programa de apoyo para elevar sus aprendizajes, es posible que afecte significativamente en el ascenso de los resultados de sus estudiantes. Con esto, se puede establecer que los docentes directivos a cargo de unidades educativas deben trabajar de manera mancomunada en 
todas las etapas de aplicación de procesos y procedimientos (implementación, monitoreo y evaluación) dirigidos a elevar los resultados de los estudiantes, debido a que esta juega un papel fundamental en contextos socioculturales vulnerables y ninguna por si solo es capaz de sustentar buenos resultados.

Se confirma al mismo tiempo, que ningún docente directivo solo o aislado de los demás puede lograr cambios significativos en los aprendizajes de los estudiantes producto de la aplicación de determinados procedimientos. Lo cual conlleva la idea de integrar a todos sus miembros en función de un objetivo y visión compartida con sentido de responsabilidad de equipo y con ánimo de colaboración más allá de la función específica de acuerdo al cargo que desempeñe dentro del establecimiento educacional.

\section{Referencias}

Cerdas, V., García, J., Torres, N., \& Fallas, M. (2017). Análisis de la gestión administrativa de centros educativos costarricenses: Percepción del colectivo docente y la dirección. Revista Ensayos Pedagógicos, 12(2), 95-122, e-ISSN: 1659-0104. Recuperado de:

\section{https://doi.org/10.15359/rep.12-2.5}

Espinosa, A. (2009). Los 9 aspectos fundamentales de la educación. Sección Columnas opinión. Colombia: Revista Dinero, publicado por el Ministerio de Educación Nacional - Mineducación. Recuperado de: https://www.mineducacion.gov.co/observatorio/1722/article210638.html

Fernández, V., Toledo, K., Martínez, E., Casado, N., Navarro, S., \& Folgado, J. (2018). Evaluación de la perspectiva de género en la docencia universitaria de la Facultad de Ciencias. Memorias del Programa de Redes-I3CE de calidad, innovación e investigación en docencia universitaria, ISBN: 978-84-09-07041-1. España: Universitat d'Alacant. Recuperado de: 
https://dialnet.unirioja.es/servlet/articulo?codigo $=6907923$ López, C. (2019). El Liderazgo y la Axiología del Docente en la Educación Primaria. Revista Scientific, 4(12), 240-253, e-ISSN: 2542-2987. Recuperado de:

https://doi.org/10.29394/Scientific.issn.2542-2987.2019.4.12.12.240$\underline{253}$

Rojas, L. (2019). Elevar el Rendimiento Académico con Estrategias Educativas. Revista Scientific, 4(12), 127-140, e-ISSN: 2542-2987. Recuperado de:

https://doi.org/10.29394/Scientific.issn.2542-2987.2019.4.12.6.127-140 Valle, G., Raiano, J., García, Suligoy, M., \& Gómez, G. (2014). Éxito y Fracaso Académico desde la Perspectiva de los Estudiantes Universitarios. Dos puntas, (9), 161-186, e-ISSN: 1852-3420. Recuperado de:

https://dialnet.unirioja.es/servlet/articulo?codigo $=5067154$ 


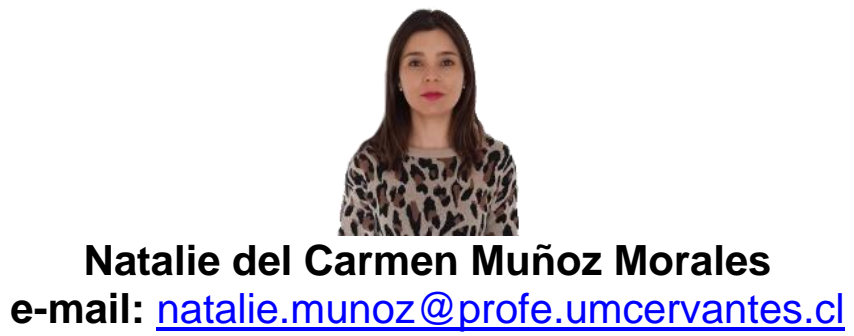

\begin{abstract}
Magister en Educación; Profesora de Ciencias mención Biología; Licenciada en Educación; Docente Académico de la Universidad Miguel de Cervantes (UMC), Santiago, Chile; Es Docente con Promoción al Investigador (PII); Ponente en Conferencias a nivel nacional e internacional; Con amplia trayectoria como académica a nivel de pregrado y postgrado; asesora de trabajos especiales de grado.

Nacida en Chile, el 4 de enero del año 1986. Doctorando en educación; Ponente en Conferencias a nivel nacional e internacional; Con amplia
\end{abstract}




\section{Albino Enzon González González}

e-mail: albino.gonzalez@profe.umcervantes.cl

Nacido en Chile, el 14 de octubre del año 1978.

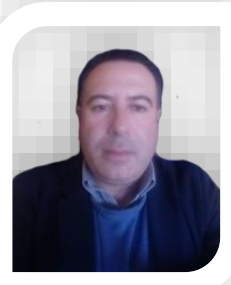

Doctorando en educación; Magister en Educación; Ingeniero en Administración; Licenciado en Ciencias de la Administración; Académico en la Dirección de Postgrado e Investigación de la Universidad Miguel de Cervantes (UMC), Santiago, Chile; Ponente en Conferencias a nivel nacional e internacional; Con amplia trayectoria como académica a nivel de pregrado y postgrado; asesor de trabajos especiales de grado. 